\begin{tabular}{|c|c|}
\hline \multirow{3}{*}{ 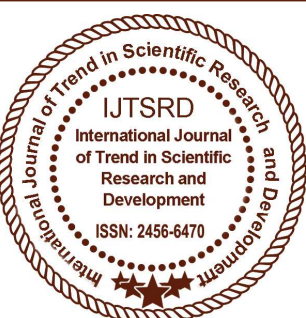 } & $\begin{array}{l}\text { International Journal of Trend in Scientific } \\
\text { Research and Development (IJTSRD) }\end{array}$ \\
\hline & International Open Access Journal \\
\hline & ISSN No: 2456 - 6470 | www.ijtsrd.com | Volume - 2 | Issue -3 \\
\hline
\end{tabular}

\title{
An Efficient Reconstructing Routing Path in Dynamic and Large scale networks using Extensive hashing
}

\author{
${ }^{1}$ A. Satish Kumar, P. Hari Priya, V. Navya, V. N. Anshitha, MD. Shabeena \\ ${ }^{1}$ Assistant Professor, \\ Department of Computer Science \& Engineering, \\ Dhanekula institute of Engineering and Technology, Vijayawada, India
}

\section{ABSTRACT}

Now-a-days, wireless sensor networks (WSNs) are getting progressively advanced with the developing system scale and the dynamic idea of remote correspondences. Numerous estimation and demonstrative methodologies rely upon per-parcel steering ways for exact and fine-grained examination of the mind boggling net-work practices. In this paper, we propose iPath, a novel way surmising way to deal with recreating the per-bundle steering ways in powerful and expansive scale systems. The fundamental thought of iPath is to misuse high way closeness to iteratively gather long ways from short ones. IPath begins with an underlying known arrangement of ways and performs way deduction iteratively. iPath incorporates a novel outline of a lightweight Extensible hashing, hash work for confirmation of the surmised ways. So as to additionally enhance the deduction ability and additionally the execution proficiency, iPath incorporates a quick bootstrapping calculation to remake the underlying arrangement of ways. We additionally execute iPath and assess its execution utilizing follows from huge scale WSN organizations and in addition broad reproductions. Results demonstrate that iPath accomplishes significantly higher remaking proportions under various system settings contrasted with other best in class approaches.

Keywords: Path inference, Wireless sensor networks, Hash function, Novel path, path similarity

\section{INTRODUCTION}

In dynamic and large scale networks, Wireless sensors are becoming increasingly complex and are applied in several application situations, e.g., structural protection, scheme management, and concrete $\mathrm{CO}$ watching.[1][3]In an exceedingly typical WSN, variety of self-organized device nodes reports the sensing information sporadically to a central sink via multichip wireless. Recent years have witnessed ascension of device network scale. Some device networks embrace lots of even thousands of device nodes. These networks typically use dynamic routing protocols to realize quick adaptation to the dynamic wireless channel conditions. The growing network scale and also the dynamic nature of wireless channel build WSNs become progressively complicated and exhausting to manage. [5]Reconstructing the routing path of every received packet at the sink aspect is efficient thanks to perceive the network's complicated internal behaviors. During this paper, we tend to propose iPath, a unique path logical thinking approach to reconstructing the per-packet routing methods in dynamic and large-scale networks. The essential plan of iPath is to take advantage of high path similarity to iteratively infer long methods from short ones.[3] iPath starts with associate degree initial notable set of methods and performs path logical thinking iteratively. iPath includes a unique style of a lightweight hash operate for verification of the inferred methods. so as to more improve the logical thinking capability likewise because the execution potency, iPath includes a quick bootstrapping algorithmic program to reconstruct the initial set of methods. [3][5]It proposes AN analytical model to calculate the booming reconstruction likelihood in varied network 
conditions like network scale, routing dynamics, packet losses, and node density. iPath achieves high reconstruction ratio in different network settings.

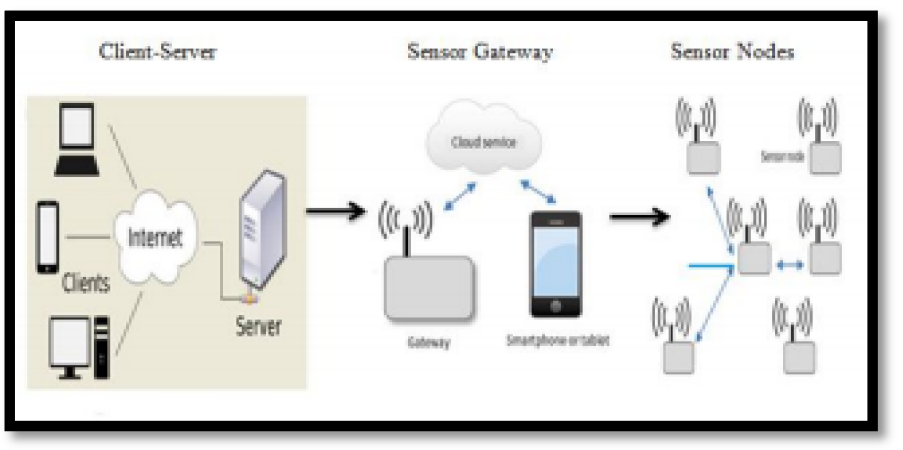

iPath wireless Sensor network

\section{LITERATURE SURVEY}

\section{A. "Wireless sensor networks: a Survey on} Environmental Monitoring"[1]

In wired IP systems, fine-grained arrange estimation incorporates numerous angles, for example, steering way recreation, bundle postpone estimation, and parcel misfortune tomography. A wireless sensor network is a group of small sensor/nodes which communicate through radio interface. These sensor nodes are composed of sensing, computation, communication and power as four basic working units. But limited energy, communication capability, storage and bandwidth are the main resource constraints. Our survey is based on various aspects of wireless sensor networks. In this paper we also discussed various types of WSNs, their applications and briefly discuss various categories of routing protocols.

\section{B. "Routing techniques in wireless sensor networks: a Survey" [5]}

In these works, tests are utilized for estimation reason.[2]-[5]Trace route is a commonplace system analytic device for showing the way numerous tests. DTrack is a test based way following framework that predicts and tracks Internet way changes. As indicated by the expectation of way changes, DTrack can track way changes viably. FineComb is a current test based system deferral and misfortune geography approach that spotlights on settling parcel reordering. Truth be told, a current work outlines the plan space of examining calculations for arrange execution estimation. Utilizing tests, be that as it may, is normally not attractive in WSNs. The primary reason is that the remote dynamic is difficult to be caught by few tests, and continuous examining will present high vitality utilization.

\section{C. "Wireless sensor networks: a Survey about Routing protocols with mobile sink" [5] [3]}

At the point when the system ends up plainly powerful, the regularly changing directing way can't be precisely reconstructed.MNT first acquires an arrangement of solid bundles from the got parcels at sink, at that point utilizes the dependable parcel set to reproduce each got parcel s path. [5] The issue of PathZip is that the pursuit space develops quickly when the system scales up. Path finder expect that all hubs produce neighborhood parcels and have a typical inter packet interim (i.e.,IPI). At that point, at the PC side, it can derive bundle ways from the compacted data. [5]-[1]Contrasted with PathZip, iPath abuses high way comparability between different bundles for quick surmising, bringing about much better adaptability. Contrasted with MNT, iPath has considerably less stringent necessities on fruitful way induction: In each jump, iPath just requires no less than one neighborhood parcel following a similar way, while MNT requires an arrangement of continuous bundles with a similar parent (called dependable parcels). Contrasted with Pathfinder, iPath does not expect regular IPI. iPath accomplishes higher recreation proportion/precision in different system conditions by misusing way closeness among ways with various lengths.

\section{EXISTING WORK}

[4]With the routing path of each packet, many measurement and diagnostic approaches are able to conduct effective management and protocol optimizations for deployed WSNs consisting of a large number of unattended sensor nodes. Path information is also important for a network manager to effectively manage a sensor network. [5]For example, given the per-packet path information, a network manager can easily find out the nodes with a lot of packets forwarded by them, i.e., network hop spots. Then, the manager can take actions to deal with that problem. Furthermore, per-packet path information is essential to monitor the fine-grained per-link metrics. For example, most existing delay and loss measurement approaches assume that the routing topology is given as a priority. [3]The timevarying routing topology can be effectively obtained by per-packet routing path, significantly improving the values of existing WSN delay and loss 
tomography approaches. [5] The main drawbacks of existing system are The growing network scale and the dynamic nature of wireless channel make WSNs become increasingly complex and hard to manage.The problem of existing approach is that its message overhead can be large for packets with long routing paths. [6]Considering the limited communication resources of WSNs, this approach is usually not desirable in practice.

\section{PROPOSED WORK}

In this paper, we propose iPath, a novel path inference approach to reconstruct routing paths at the sink side. [1]Based on a real-world complex urban sensing network with all node generating local packets, we find a key observation: It is highly probable that a packet from node and one of the packets from one's parent will follow the same path starting from another's parent toward the sink. We refer to this observation as high path similarity.[2]The basic idea of iPath is to exploit high path similarity to iteratively infer long paths from short ones. iPath starts with a known set of paths and performs path inference iteratively. During each iteration, it tries to infer paths one hop longer until no paths can be inferred.In order to ensure correct inference, iPath needs to verify whether a short path can be used for inferring a long path. For this purpose, iPath includes a novel design of a lightweight hash function. Each data packet attaches a hash value that is updated hop by hop. This recorded hash value is compared against the calculated hash value of an inferred path. If these two values match, the path is correctly inferred with a very high probability.[4]In order to further improve the inference capability as well as its/execution efficiency, iPath includes a fast bootstrapping algorithm to reconstruct a known set of paths.

\section{A. Iterative Bootstrapping algorithm:}

IPath reconstructs unknown long paths from known brief paths iteratively. By means of evaluating the recorded direction worth and the calculated course price, the sink can confirm whether or not a protracted course and a short direction share the identical direction after the quick course's usual node. [1]The Iterative-Boosting method involves the major common sense of the algorithm that tries to reconstruct as many as possible packets iteratively.[2] The center is an initial set of packets whose paths were reconstructed and a collection of other packets. For the period of every iteration, is a set of newly reconstructed packet paths. The algorithm tries to make use of each and every packet in to reconstruct every packet's route in community topologies. The procedure ends when no new paths will also be reconstructed viable paths.

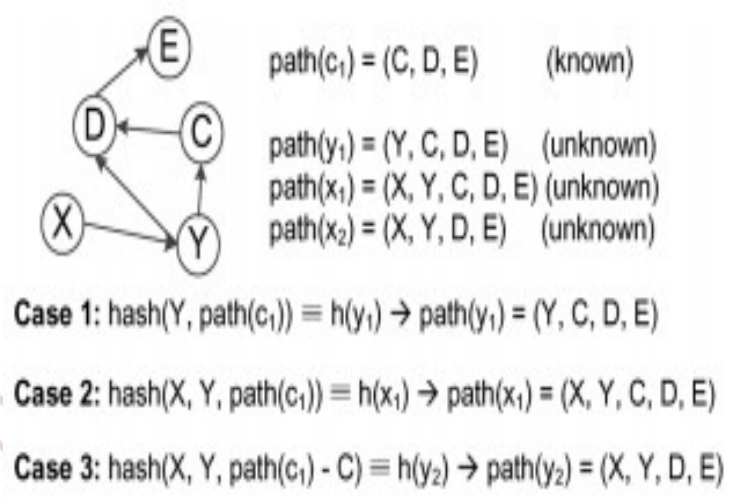

\section{B. Fast Bootstrapping Algorithm:}

[1]The unvaried boosting algorithmic rule desires associate initial set of reconstructed ways. Additionally to the one/two-hop ways, the quick bootstrapping algorithmic rule any provides additional initial reconstructed ways for the unvaried boosting algorithmic rule. These initial reconstructed ways cut back the amount of iterations required and speed up the unvaried boosting algorithmic rule.[1]-[3] The basic idea is to reconstruct a packet's path by the help of the local packets at each hop. For each node, we can obtain its stable periods by the parent change counter attached in each of its header.

\section{Extensive Hashing:}

[3]The usage of a square table to depict bits of the hash being utilized to the limit pieces is the key idea. You can reuse ruins until the point that they are full. Pieces simply should be part when they finish off.

The basic idea of Extensive hashing is to ensure correct inference, iPath needs to verify whether a short path can be used for inferring a long path. For this purpose, iPath includes a novel design of a lightweight hash function. Each data packet attaches a hash value that is updated hop by hop. This recorded hash value is compared against the calculated hash value of an inferred path. If these two values match, the path is correctly inferred with a very high probability. 


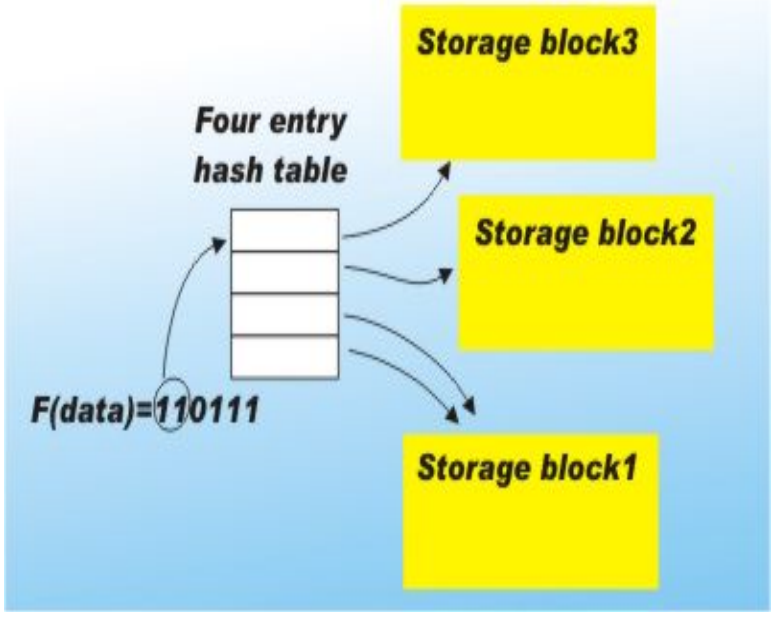

\section{FUTURE ENHANCEMENT}

Our destiny paintings consists of to examine our proposed WSN dynamic routing topology inference with incomplete direction dimension set in a collection cycle because of packet loss in actual-world environments. We plan to further look into our WSN dynamic routing topology inference method for massive-scale of WSNs including hundreds of nodes. We also plan to enforce the proposed approach and check it very well in a real-international WSN check mattress. Based totally on the dynamic topology inference, modern WSN link loss and de-s lay inference schemes can be prolonged to deal with sensible WSNs below dynamic routing.

\section{REFERENCES}

1. www.ijmetmr.com/oljune2017/MSoumyaGBhagyaLakshmi-69.pdf Wireless sensor networks path inference:iPath

2. http://ieeexplore.ieee.org/document/6977988/ iPath:path inference in wireless sensor networks by Yi.Gao in 2016.

3. Z. Li, M. Li, and Y. Liu, "Towards energy fairness in asynchronous duty-cycling sensor networks," Trans. Sensor Networks., vol. 10, no. 3, pp. 38:1-38:26, 2014.

4. Y.Gaoet al., "iPath: Path inference in wireless sensor networks,” Tech. Rep., 2014 [Online]. Available: http://www.emnets.org/pub/gaoyi/techipath.pdf

5. Y. Liang and R. Liu, "Routing topology inference for wireless sensor networks," Comput. Commun. Rev., vol. 43, no. 2, pp. 21-28, 2013.

\section{CONCLUSION}

In this paper, we propose iPath, a novel path inference approach for reconstructing the routing path for each received packet. iPath exploits the path similarity and uses the iterative boosting algorithm to reconstruct the routing path effectively. This bootstrapping algorithm provides an initial set of paths for the iterative algorithm. This iPath achieves high path similarity in dynamic networks. The results show that iPath achieves higher reconstruction ratio in different network settings. 